\title{
Vertrauen ist gut, Kontrolle ist besser?
}

\section{Dr. med. Yvonne Gilli}

Mitglied des FMH-Zentralvorstandes, Departementsverantwortliche Digitalisierung/eHealth

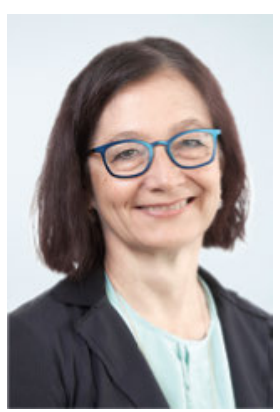

Der Fall Facebook und Cambridge Analytics hat uns in eindrücklicher Weise vor Augen geführt, in welchem Umfang persönliche Daten in einem komplizierten Netz von Datenhändlern illegal und manipulativ entwendet werden können. Anfang März wurde bekannt, dass im Jahr 2014 Daten von 50 Millionen FacebookNutzern ohne deren Einwilligung systematisch erfasst und ausgewertet wurden, um Profile nach sexueller Orientierung oder politischen Interessen zu erstellen. Zwar hat Facebook bereits 2015 die Löschung der Daten durchgesetzt, aber eine nachträgliche Überprüfung des Falls fand nicht statt.

\section{Das Vertrauen in die Datensicherheit ist entscheidend für die Akzeptanz des elektro- nischen Patientendossiers.}

Dass die Angst vor Datenmissbrauch hoch ist, zeigt eine im Januar 2017 durchgeführte Studie von Comparis, bei der 1000 Personen nach ihrem Vertrauen in Online-Dienste befragt wurden. ${ }^{1}$ Obwohl das Vertrauen in Social-Media-Plattformen mit etwa $12 \%$ gering ist, stieg der Umsatz von Facebook im Jahr 2017 um mehr als $40 \%$ und täglich loggen sich mehr als 1 Milliarde Menschen mindestens einmal am Tag ein. Offenbar schätzen Nutzer den individuellen Nutzen höher ein als die Gefahr, die von einer Datenweitergabe ausgeht.

In der diesjährigen Befragung des Swiss eHealth Barometers wurden Ärztinnen und Ärzte sowie Bürgerinnen und Bürger über ihr Vertrauen in die Einhaltung des Datenschutzes im Zusammenhang mit dem elek-

1 https://www.comparis. ch/comparis/press/ medienmitteilungen/ artikel/2017/digital/ datenvertrauensindex-2017/ datenvertrauensindex 2 Zitat von Aurélie Rosemberg, Artikel 24heures vom 29.3.2018. tronischen Patientendossier (EPD) befragt. 16\% der befragten Bürger vertrauen den Stellen, welche mit den Patientendaten arbeiten, voll und ganz, dass sie den Datenschutz rund um das EPD einhalten. Dahingegen haben $49 \%$ nur ein tendenzielles Vertrauen. Die Ärzteschaft hingegen ist eher kritischer eingestellt: Nur $4 \%$ der befragten Ärzte haben volles Vertrauen und 46\% ein tendenzielles Vertrauen. Im Umkehrschluss bedeutet das, dass $46 \%$ der Ärztinnen und Ärzte den Stellen eher weniger oder überhaupt nicht vertrauen. Ihre Wahrnehmung des Sicherheitsaspektes spielt für die Entscheidung, ob sie für oder gegen das EPD sind, eine wichtige Rolle.

In den letzten Tagen war zu lesen, dass die Westschweizer Kantone sich in der interkantonalen Vereinigung Cara zusammengeschlossen haben, die das Ziel verfolgt, die Entwicklung des EPD in der Westschweiz voranzutreiben. Zuletzt hat sich der Kanton Wallis zu einem Beitritt entschlossen. Dabei werden Erinnerungen an das Jahr 2015 wach, als bekannt wurde, dass das Walliser Patientendossier «Infomed" den Dienst Google Analytics verwendet, um seine Zahlen und Nutzerdaten zu analysieren - notabene ohne die Nutzer zu informieren. Daneben wurde noch über angebliche Sicherheitslücken berichtet. Im Fall Cara ist man zuversichtlicher, da die Systeme regelmässig auditiert würden und es somit keine Datenschutzverletzungen gebe («Aucun problème de sécurité des données n'est à déplorer $\left.{ }^{2} »\right)$. Eine Zertifizierung und eine jährliche Audi-

\section{Der Fall «Cambridge Analytics» zeigt, dass die Einhaltung von Datenschutzvereinbarungen überprüft werden muss.}

tierung von Gemeinschaften, wie sie im Bundesgesetz über das elektronische Patientendossier vorgesehen ist, kann jedoch nicht sicherstellen, dass die gesetzlichen Bestimmungen im Einzelfall tatsächlich auch eingehalten werden. Die Verpflichtung liegt also bei den $\mathrm{Ge}$ meinschaften, welche laut Verordnung als sicherheitsrelevant eingestufte Vorfälle dem Bundesamt für Gesundheit melden müssen. Vertrauen ist also gut und Kontrolle ist bekanntlich besser. Aber die entscheidende Frage lautet: Wer übt eigentlich die Kontrollfunktion aus, wenn es um das elektronische Patientendossier geht? 